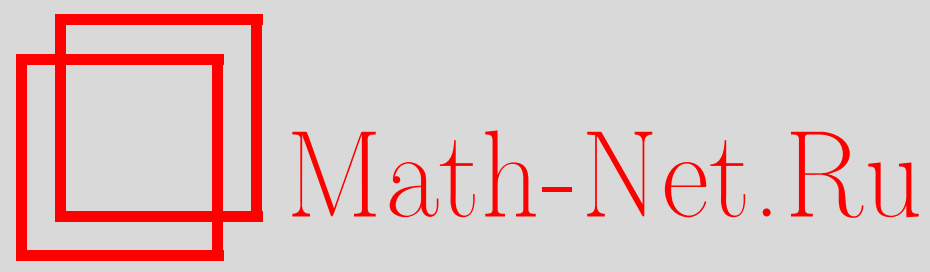

А. И. Дегтярев, В. И. Звонилов, Жесткая изотопическая классификация вещественных алгебраических кривых бистепени $(3,3)$ на квадриках, Матем. заметки, 1999, том 66, выпуск 6, 810-815

DOI: https://doi.org/10.4213/mzm1226

Использование Общероссийского математического портала Math-Net.Ru подразумевает, что вы прочитали и согласны с пользовательским соглашением http://www . mathnet.ru/rus/agreement

Параметры загрузки:

IP : 3.85 .5 .30

26 апреля 2023 г., $17: 03: 26$ 


\title{
ЖЕСТКАЯ ИЗОТОПИЧЕСКАЯ КЛАССИФИКАЦИЯ ВЕЩЕСТВЕННЫХ АЛГЕБРАИЧЕСКИХ КРИВЫХ БИСТЕПЕНИ $(3,3)$ НА КВАДРИКАХ
}

\section{А. И. Дегтярев, В.И. Звонилов}

\begin{abstract}
Жесткой изотопией неособых вещественных алгебраических кривых данной бистепени на квадрике называется путь в пространстве таких кривых. В работе получена жесткая изотопическая классификация неособых вещественных алгебраических кривых бистепени $(3,3)$ на гиперболоиде и эллипсоиде. Перечислены также компоненты связности пространства вещественных алгебраических кривых бистепени $(3,3)$, имеющих единственную невырожденную двойную точку или точку возврата.

Библиография: 11 названий.
\end{abstract}

1. Введение. Понятие жесткой изотопии было введено Рохлиньм [1]. На проективной плоскости для неособых вещественных алгебраических кривых степени $m$ классификация с точностью до жестких изотопий известна для $m \leqslant 6$ (см. [1]-[3]). На квадриках жесткие изотопии вещественных алгебраических кривых бистепеней $(m, 1)$, $(m, 2)$ изучались авторами [4], [5]; при этом для неособьх кривых они получили жесткую изотопическую классификацию. Для (неособых) кривых бистепени $(3,3)$ на квадриках классификация их вещественных схем (т.е. вещественная изотопическая классификация) была получена в работах [6], [7] (см. также [8]), а классификация их комплексных схем (вещественных схем, наделенных типом и комплексными ориентациями, см. ниже п. 2) - в [8]. В настояшей работе мы доказьваем, что неособая кривая бистепени $(3,3)$ на гиперболоиде (на эллипсоиде) определяется с точностью до жесткой изотопии своей вещественной (комплексной) схемой (теорема 2). В доказательстве теоремы 1 мы указьваем все компоненты связности пространства кривых бистепени $(3,3)$, имеющих единственную невырожденную двойную точку или точку возврата (см. рис. 1, 2). Мы используем подход, предложенный в [9] для получения жесткой изотопической классификации плоских вещественных квартик.

2. Определения и обозначения. Пусть $X$ - неособая квадрика. Комплексной частью $\mathbb{C} X$ поверхности $X$ является $\mathbb{C} P^{1} \times \mathbb{C} P^{1}$, и на $X$ можно ввести две, с точностью до биголоморфизма, антиголоморфные инволюции с непустой вещественной частью; в результате получаются две вещественные поверхности: гиперболоид с вещественной частью $\mathbb{R} X$, гомеоморфной тору, и эллипсоид с $\mathbb{R} X \cong S^{2}$.

Работа второго автора выполнена при поддержке Министерства образования России (конкурс на базе С-ПбГУ), грант № 97-0-1.2-41. 
Зафиксируем пару $P_{1}, P_{2}$ образующих квадрики $X$. Фундаментальные классы $\left[\mathbb{C} P_{1}\right]$, $\left[\mathbb{C P}_{2}\right]$ образуют базис группы $H_{2}(\mathbb{C} X) \cong \mathbb{Z} \oplus \mathbb{Z}$. Пусть $A$ - алгебраическая кривая на $X$. Тогда $[\mathbb{C} A]=m_{1}\left[\mathbb{C} P_{1}\right]+m_{2}\left[\mathbb{C} P_{2}\right]$ для некоторых неотрицательных целых $m_{1}, m_{2}$. Пара $\left(m_{1}, m_{2}\right)$ назьвается бистепенью кривой $A$. Если $\left[x_{0}: x_{1}\right],\left[y_{0}: y_{1}\right]$ - однородные координаты на прямых $P_{1}, P_{2}$, то кривая $A$ задается биоднородньм многочленом

$$
F\left(x_{0}, x_{1} ; y_{0}, y_{1}\right)=\sum_{i, j=1}^{m_{1}, m_{2}} a_{i j} x_{1}^{i} x_{0}^{m_{1}-i} y_{1}^{j} y_{0}^{m_{2}-j}
$$

имеюшим степени однородности $m_{1}$ по $x_{0}, x_{1}$ и $m_{2}$ по $y_{0}, y_{1}$.

На гиперболоиде антиголоморфная инволюция conj переводит каждую из четырех координат в комплексно сопряженную; поэтому кривая $A$ вешественна тогда и только тогда, когда все $a_{i j}$ вещественны. На эллипсоиде инволюция conj действует по формуле $\left(\left[x_{0}: x_{1}\right],\left[y_{0}: y_{1}\right]\right) \mapsto\left(\left[\bar{y}_{0}: \bar{y}_{1}\right],\left[\bar{x}_{0}: \bar{x}_{1}\right]\right)$, и вещественность кривой $A$ означает, что $a_{i j}=\bar{a}_{j i}$ (в частности, $\left.m_{1}=m_{2}\right)$.

Для указания топологии вещественной кривой на квадрике мы используем модификацию стандартной кодировки вещественных схем плоских проективных кривых (см., например, [10]). Пусть сначала $(X, \mathrm{conj})$ - гиперболоид и $A \subset X$ - неособая вещественная алгебраическая кривая. Вещественная часть $\mathbb{R} A$ может иметь компоненты двух типов: стягиваемые в $\mathbb{R} X$ и нестягиваемые; стягиваемые компоненты называются ов $а$ лами. Число овалов обозначается через $l$, число нестягиваемых компонент - через $h$. Каждый овал ограничивает топологический диск в $\mathbb{R} X$, назьваемый внутренностью овала. Фундаментальные классы $\left[\mathbb{R} P_{1}\right],\left[\mathbb{R} P_{2}\right]$, наделенные некоторыми (фиксированными) ориентациями, образуют базис группы $H_{1}(\mathbb{R} X) \cong \mathbb{Z} \oplus \mathbb{Z}$. Все нестягиваемые компоненты $N_{1}, \ldots, N_{h}$ реализуют один и тот же ненулевой класс $\left(c_{1}, c_{2}\right)$ в $H_{1}(\mathbb{R} X)$, где $c_{1}, c_{2}$ взаимно просты. Вешественная схема кривой $\mathbb{R} A \subset \mathbb{R} X$ кодируется следуюшим образом:

$$
\left\langle\left(c_{1}, c_{2}\right), \text { scheme }_{1},\left(c_{1}, c_{2}\right), \text { scheme }_{2}, \ldots,\left(c_{1}, c_{2}\right), \text { scheme }_{h}\right\rangle,
$$

где scheme $_{1}, \ldots$, scheme $_{h}$ - схемы расположения овалов, лежащих в связных компонентах поверхности $\mathbb{R} X \backslash\left(N_{1} \cup \cdots \cup N_{h}\right)$ (ср. с [10], [8]).

Если $(X$, conj $)$ - эллипсоид, то все компоненты кривой $\mathbb{R} A$ являются овалами; их число обозначается через $l$. В этом случаемы фиксируем точку $\infty \in \mathbb{R} X \backslash \mathbb{R} A$, называемую внешней, и для овала $C \subset \mathbb{R} X$ определяем его внутренность как компоненту поверхности $\mathbb{R} X \backslash C$, не содержащую $\infty$. При этом на множестве овалов возникает естественный частичньй порядок, и схема расположения овалов кодируется так же, как в [10].

Согласно $\Phi$. Клейну (см. [11] или [1]) вещественная кривая $A$ принадлежит тuпy I или muny II в зависимости от того, разбивает $\mathbb{R} A$ комплексификацию $\mathbb{C} A$ или нет. Если $A$ принадлежит типу I, естественные ориентации компонент $U$ и $V$ пространства $\mathbb{C} A \backslash \mathbb{R} A$ дают две противоположные ориентации кривой $\mathbb{R} A=\partial U=\partial V$; они назьваются комплексными ориентациями. Вещественная схема, наделенная типом и, в случае типа I, комплексными ориентациями, называется комплексной схемой. Если нужно указать тип кривой с вешественной схемой $\langle B\rangle$, используются обозначения $\langle B\rangle_{\mathrm{I}}$ и $\langle B\rangle_{\mathrm{II}}$.

Все вещественные кривые бистепени $(m, n)$ образуют пространство $C_{m, n} \cong \mathbb{R P}^{N}$ с $N=m n+m+n$. Множество $\Delta \subset C_{m, n}$ особых кривых имеет размерность $N-1$. Обозначим через $S \subset \Delta$ подмножество кривых, имеющих особую точку, отличную от 
невырожденной двойной точки или точки возврата, или имеющих несколько особых точек. Множество $\Delta \backslash S$ является топологическим многообразием (хотя и не гладким подмногообразием в $\left.C_{m, n}\right)$. Жестким изотопическим классом кривой $A \in C_{m, n} \backslash \Delta$ (или $A \in \Delta \backslash S$ ) назьвается компонента пространства $C_{m, n} \backslash \Delta($ соответственно $\Delta \backslash S)$, содержашая $A$. Компоненты пространства $C_{m, n} \backslash \Delta($ соответственно $\Delta \backslash S)$ назьваются камерами (стенками).

3. Кривые с невырожденной двойной точкой или точкой возврата. Сначала мы перечислим стенки в $C_{3,3}$.

Теорема 1. Число стенок в $C_{3,3}$ равно 20 в случае гиперболоида и 6 в случае әллипсоида.

ДокАЗАТЕЛЬСтво. Возьмем кривую $A \in \Delta \backslash S$, раздуем ее особую точку и стянем две образующие, проходящие через эту точку. В результате получим неособую квартику $Q \subset \mathbb{P}^{2}$. Обратное преобразование задается парой $\left(q_{1}, q_{2}\right)$ различных точек на $Q$ (образов образующих), вещественных в случае гиперболоида и комплексно сопряженных в случае эллипсоида. В случае гиперболоида эти точки упорядочены и вещественная прямая, проходящая через них, ориентирована. Если $A$ имеет точку возврата, эта прямая является касательной к кривой $Q$.

Итак, в случае эллипсоида ясно, что стенки нумеруются жесткими изотопическими классами вещественных квартик. Согласно [11] число этих классов равно 6.

В случае гиперболоида стенки нумеруются компонентами связности пространства Conf конфигураций $\left(Q, q_{1}, q_{2}, \varepsilon\right)$, где $\varepsilon$ - ориентация вещественной прямой, проходящей через $q_{1}$ и $q_{2}$. Поэтому вместе с жестким изотопическим типом квартики $Q$ мы должны учитывать различные расположения точек $q_{1}$ и $q_{2}-$ на одном и том же или на разных овалах кривой $Q$. В последнем случае обозначим конфигурацию $\left(Q, q_{1}, q_{2}, \varepsilon\right)$ через $b_{\alpha}$, $\alpha=2,3,4$, если вешественная схема кривой $Q$ есть $\langle\alpha\rangle$ (т.е. состоит из $\alpha$ овалов, лежащих вне друг друга), и через $b_{\text {inn }}$ или $b_{\text {out }}$, если она является әнездом $\langle 1\langle 1\rangle\rangle$ (т.е. состоит из двух овалов, один из которых лежит внутри другого) и точка $q_{1}$ принадлежит внутреннему или соответственно внешнему овалу гнезда. В первом случае обозначим эту конфигурацию через $a_{\alpha}, \alpha=1, \ldots, 4, a_{\text {inn }}$ или $a_{\text {out }}$ соответственно.

Кроме того, все конфигурации за исключением $b_{2}, b_{3}$ снабжаются зна́ком "+” или "-" следующим образом. Точки $q_{1}, q_{2}$ разделяют вещественную прямую, проходящую через них, на два отрезка, ориентированных в согласии с $\varepsilon$; пусть $\overline{q_{1} q_{2}}-$ тот из них, начало которого есть $q_{1}$. Обозначим конфигурацию $\left(Q, q_{1}, q_{2}, \varepsilon\right)$ через $a_{\alpha}^{-}$(соответственно через $\left.b_{\text {inn }}^{-}, b_{\text {out }}^{-}\right)$, если объединение отрезка $\overline{q_{1} q_{2}}$ с овалом, содержащим точки $q_{1}, q_{2}$ (соответственно с внешним овалом гнезда), имеет ориентируемую окрестность в $\mathbb{R} P^{2}$, и через $a_{\alpha}^{+}\left(\right.$соответственно через $b_{\text {inn }}^{+}, b_{\text {out }}^{+}$) в противном случае. Для $b_{4}$ рассмотрим в $\mathbb{R} P^{2}$ одностороннюю топологическую окружность $\gamma$, которая не пересекает $\mathbb{R} Q \cup \overline{q_{1} q_{2}}$, и обозначим конфигурацию через $b_{4}^{-}$(соответственно через $b_{4}^{+}$), если два овала, содержащих $q_{1}$ и $q_{2}$, имеют одинаковые (соответственно различные) комплексные ориентации в $\mathbb{R} P^{2} \backslash \gamma$.

Для завершения доказательства остается заметить, что множество компонент связности пространства Conf находится в естественном взаимно однозначном соответствии с множеством $\left\{a_{\alpha}^{ \pm}(\alpha=1, \ldots, 4), a_{\text {inn }}^{ \pm}, a_{\text {out }}^{ \pm}, b_{2}, b_{3}, b_{4}^{ \pm}, b_{\text {inn }}^{ \pm}, b_{\text {out }}^{ \pm}\right\}$, состоящим из 20 әлементов. Это утверждение вытекает, очевидно, из следующей леммы. 
ЛЕММА О ПЕРЕСТАНОВКЕ ОВАЛОВ. ДЛя любой отличной от әнезда вещественной квартики $Q$ существует жсесткая изотопия, переводящая $Q$ в себя и индуцирующая любую заданную перестановку овалов кривой $Q$. При этом если $Q$ состоит из двух или трех овалов, существует жесткая изотопия, переводящая каждый овал в себя и обращающая ориентащию заданной прямой, пересекающей два овала.

ДокАЗАТЕЛЬСТво. Так как жесткий изотопический класс неособой вещественной квартики определяется ее вещественной схемой (см. [11] или [1]) и групп $\operatorname{PGL}(3 ; \mathbb{R})$ связна, достаточно реализовать каждую вещественную схему $\langle\alpha\rangle, \alpha=2,3,4$, некоторой квартикой $Q$ и найти для нее проективные преобразования, индуцирующие требуемые жесткие изотопии. Для $\alpha=2$ в качестве $Q$ можно взять кривую

$$
\left(4 x_{1}^{2}+4 x_{2}^{2}-x_{0}^{2}\right)\left(4 x_{0}^{2}+4 x_{2}^{2}-x_{1}^{2}\right)=t\left(x_{0}^{4}+x_{1}^{4}+x_{2}^{4}\right),
$$

где $t \in \mathbb{R}$ достаточно мало. Тогда преобразование $\left(x_{0}, x_{1}, x_{2}\right) \mapsto\left(x_{1},-x_{0}, x_{2}\right)$ переставляет овалы кривой $Q$. Для $\alpha=3$ и $\alpha=4$ рассмотрим куб $\left\{\left(x_{0}, x_{1}, x_{2}\right):\left|x_{i}\right| \leqslant 1\right\}$ в $\mathbb{R}^{3}$. Пусть $u\left(x_{0}, x_{1}, x_{2}\right)=0$ - уравнение, задающее объединение четырех плоскостей, проходящих через начало координат и пересекающих этот куб по правильным шестиугольникам. Тогда уравнение $u\left(x_{0}, x_{1}, x_{2}\right)+t\left(x_{0}^{4}+x_{1}^{4}+x_{2}^{4}\right)=0$ при достаточно малом $t$ дает неособую квартику $Q \subset \mathbb{P}^{2}$. В зависимости от знака числа $t$ эта квартика имеет 4 или 3 овала; каждьй из них соответствует паре противоположных вершин или соответственно граней куба. Поэтому любая заданная перестановка овалов осуществляется некоторой симметрией куба. Кроме того, для $\alpha=2$ и $\alpha=3$ преобразование $\left(x_{0}, x_{1}, x_{2}\right) \mapsto\left(x_{0},-x_{1}, x_{2}\right)$ переводит каждый овал в себя и меняет ориентацию прямой $x_{2}=0$.

\section{4. Основной результат.}

Теорема 2. Жесткая изотопическая классификация неособых вещественных алгебраических кривых бистепени $(3,3)$ на гиперболоиде (на эллипсоиде) совпадает с классификацией их комплексных (вещественных) схем. Графы примыканий камер в $C_{3,3}$ показаны на рис. 1 (для гиперболоида) и на рис. 2 (для әллипсоида).

ДокАЗАТЕЛЬСтво. Рассмотрим точную последовательность тройки $\left(C_{3,3}, \Delta, S\right)$ (напомним, что $\operatorname{dim} C_{3,3}=15$ )

$$
0 \rightarrow H_{15}\left(C_{3,3}, S\right) \rightarrow H_{15}\left(C_{3,3}, \Delta\right) \rightarrow H_{14}(\Delta, S) \stackrel{\text { in }}{\rightarrow} H_{14}\left(C_{3,3}, S\right)
$$

(здесь и ниже все гомологии с $\mathbb{Z} / 2$-коэффициентами). Ясно, что $c$ - число камер - равно $\operatorname{dim}_{\mathbb{Z} / 2} H_{15}\left(C_{3,3}, \Delta\right)$, а $w$ - число стенок - равно $\operatorname{dim}_{\mathbb{Z} / 2} H_{14}(\Delta, S)$. Поскольку $H_{15}\left(C_{3,3}, S\right)=H_{15}\left(C_{3,3}\right)=\mathbb{Z} / 2$, из точности последовательности (1) следует, что

$$
c=1+w-\operatorname{codim}_{\mathbb{Z} / 2} \text { ker in } .
$$

В случае эллипсоида, поскольку $w=6$ по теореме $1, c \leqslant 7$. С другой стороны, возмущая соответствующие особые кривые, получаем 7 камер, различающихся вещественным схемами. Это завершает доказательство для эллипсоида. 


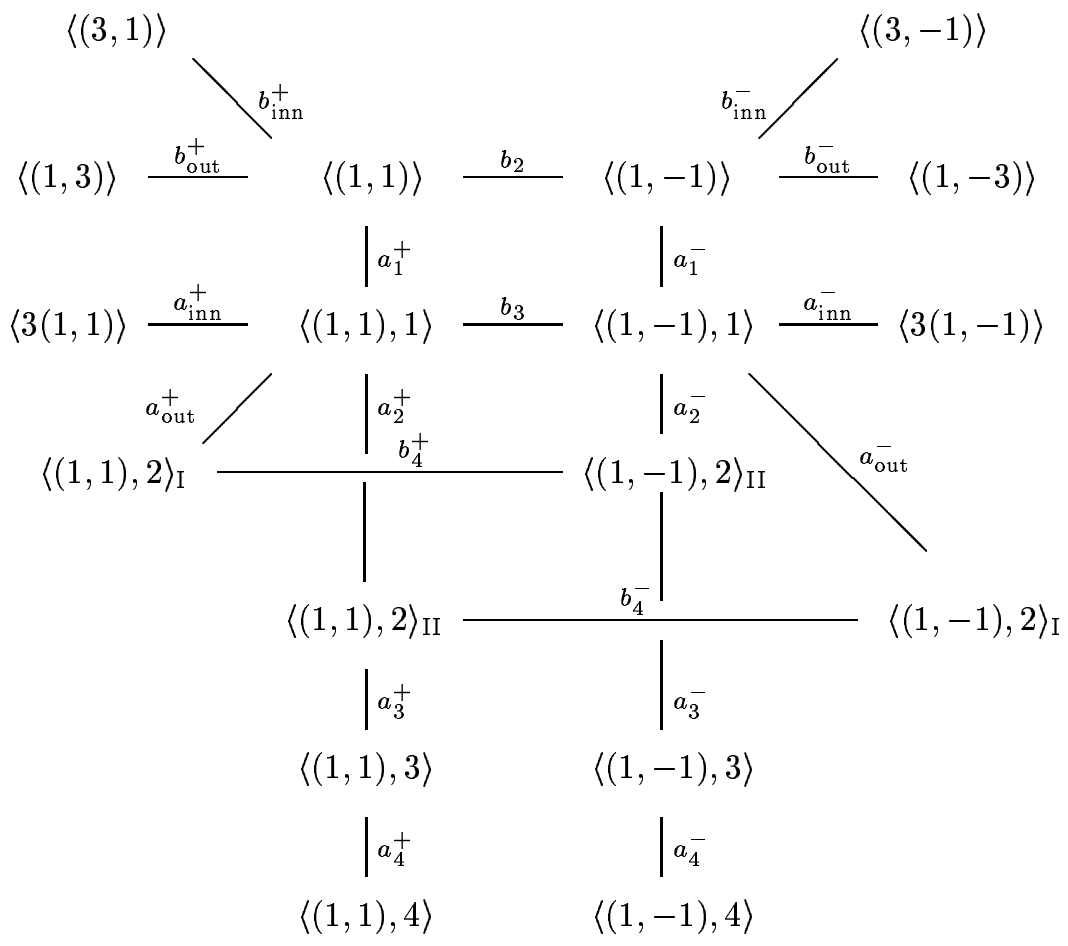

Рис. 1. Камеры и стенки пространства кривых бистепени $(3,3)$ на гиперболоиде

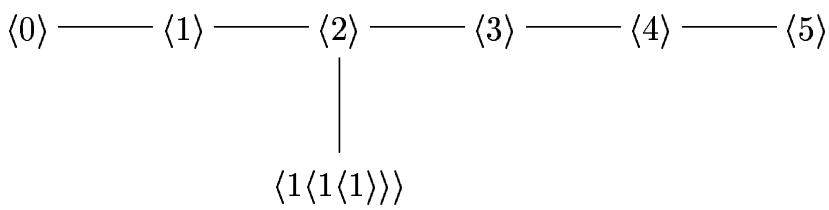

Р ис. 2. Камеры пространства кривых бистепени $(3,3)$ на эллипсоиде

В случае гиперболоида число комплексных схем кривых бистепени $(3,3)$ равно 18 (см. $[8$, п. 3.10$] ;$ заметим, что в [8] комплексные схемы изучались с точностью до перестановки сомножителей произведения $\mathbb{P}^{1} \times \mathbb{P}^{1}$, поэтому их число было равно 9 ). Следовательно, $c \geqslant 18$. Поскольку $w=20$ (теорема 1 ), для получения противоположного неравенства достаточно доказать, что $\operatorname{codim}_{\mathbb{Z} / 2}$ ker in $\geqslant 3$. Классы $w_{j}$, реализуемые стенками, очевидно, образуют базис пространства $H_{14}(\Delta, S)$. Пусть $x_{j}$ - координаты класса $x \in H_{14}(\Delta, S)$ в этом базисе. Тогда $\operatorname{codim}_{\mathbb{Z} / 2}$ ker in есть число линейно независимых уравнений, которые в координатах $x_{j}$ задают ker in. Поэтому достаточно найти три таких уравнения. Согласно двойственности Александера-Понтрягина

$$
H_{14}\left(C_{3,3}, S\right) \cong H^{1}\left(C_{3,3} \backslash S\right)=\operatorname{Hom}\left(H_{1}\left(C_{3,3} \backslash S\right), \mathbb{Z} / 2\right) .
$$

Следовательно, $x \in$ ker in тогда и только тогда, когда in $x \circ H_{1}\left(C_{3,3} \backslash S\right)=0$, и нужные уравнения получаются умножением равенства in $x=\sum x_{j}$ in $w_{j}$ на некоторые линейно 
независимые классы из $H_{1}\left(C_{3,3} \backslash S\right)$. Эти классы задаются маленькими окружностями в $C_{3,3} \backslash S$, центры которых лежат в $S$ и соответствуют трем кривым, указанным на рис. 3-5 (кривые на рис. 4 и 5 различаются комплексньми ориентациями). Эти кривые легко строятся возмущением объединения прямых. Каждая окружность пересекает только четыре стенки, причем каждую трансверсально в одной точке. Поэтому в силу (2) реализуемые этими окружностями классы линейно независимы, а получаемые с их помощью уравнения ненулевые.

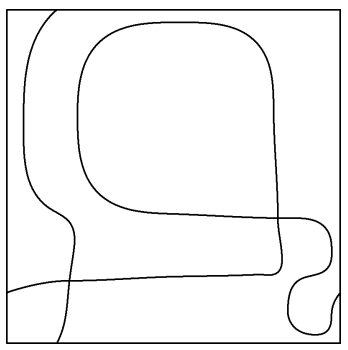

Рис. 3

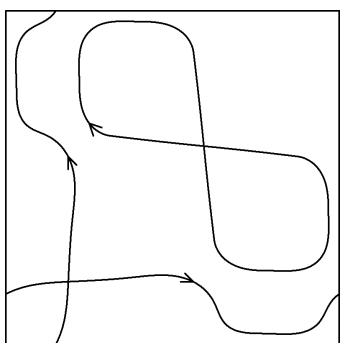

Рис. 4

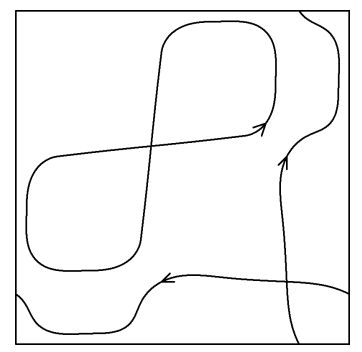

Рис. 5

\section{СПИСОК ЦИТИРОВАННОЙ ЛИТЕРАТУРЫ}

[1] Рохлин В. А. Комплексные топологические характеристики вещественных алгебраических кривых // УМН. 1978. Т. 33. № 5. С. 77-89.

[2] Харламов В.М.Жесткая изотопическая классификация вещественных плоских кривых степени 5 // Функцион. анализ и его прилож. 1981. Т. 15. №1. С. 88-89.

[3] Никулин В.В.Целочисленные квадратичные формы и некоторые их геометрические приложения // Изв. АН СССР. Сер. матем. 1979. Т. 43. №1. С. 111-177.

[4] Degtyarev A. I. Appendix // Kharlamov V. M., Rokhlin V. A., Viro O. Ya. Topological properties of real plane projective algebraic curves (to appear).

[5] Zvonilov V.I. Stratified spaces of real algebraic curves of bidegree $(m, 1)$ and $(m, 2)$ on a hyperboloid // Amer. Math. Soc. Transl. Ser. 2. 1996. V. 173. P. 253-264.

[6] Гудков Д.А., Усачев А.К. Неособые кривые младших порядков на гиперболоиде // Методы качественной теории дифференциальных уравнений. Горький, 1980. С. 96-103.

[7] Hilbert D. Ueber die reelen Zuge algebraischen Curven // Math. Ann. 1891. V. 38. P. 115-138.

[8] Звонилов В. И. Комплексные топологические инварианты вещественньх алгебраических кривых на гиперболоиде и эллипсоиде // Алгебра и анализ. 1991. Т. 3. № 5. С. 88-108.

[9] Kharlamov V. M., Rokhlin V.A., Viro O. Ya. Topological properties of real plane projective algebraic curves (to appear).

[10] Виро О.Я. Успехи в топологии вещественных алгебраических многообразий за последние шесть лет // УМН. 1986. Т. 41. №3. С. 45-67.

[11] Klein F. Gesammelte mathematische Abhandlungen. V. 2. Berlin, 1922.

Bilkent University (Анкара, Турция)

Поступило

Ськтывкарский государственный университет

04.11 .98

E-mail : degt@fen.bilkent.edu.tr,

Исправленный вариант

zvonilov@ssu.edu.komi.ru 